\title{
Fetal Onset of General Movements
}

\author{
ANNEMARIE B. LÜCHINGER, MIJNA HADDERS-ALGRA, COLETTE M. VAN KAN, AND JOHANNA I. P. DE VRIES
}

\begin{abstract}
Department of Obstetrics and Gynaecology [A.B.L., J.I.P.V.], VU University Medical Centre, 1007 MB, Amsterdam, The Netherlands; Department of Neurology [M.H.-A.], University Medical Centre Groningen, 9700 RB Groningen, The Netherlands; Department of Farm Animal Health [C.M.K.], Utrecht University, 3508 TD, Utrecht, The Netherlands
\end{abstract}

\begin{abstract}
Perinatal qualitative assessment of general movements (GMs) is a tool to evaluate the integrity of the young nervous system. The aim of this investigation was to study the emergence of GMs. Fetal onset of GMs was studied sonographically in 18 fetuses during the first trimester of uncomplicated in vitro fertilization (IVF) pregnancies in weekly assessments. The earliest motility consisting of small and simple sideways bending (SB) of head and/or rump starts at $7 \mathrm{wk}$, lasting about $1 \mathrm{~s}$. Between 7 and $8.5 \mathrm{wk}$, motility differentiates further into movements in which also one or two arms or legs become active; movements are still slow, small, and in one direction, but the duration increases to a few seconds. The transition into GMs at $9-10 \mathrm{wk}$ is characterized by variation in participating body parts and amplitude, speed, and direction during longer periods of time. Between 9 and $13 \mathrm{wk}$, simple and stereotyped SBs and GMs may coexist. At $9 \mathrm{wk}$, the incidence of SBs decreases $(p=0.01)$ and that of GMs increases $(p=0.006)$. The data suggest that initial simple fetal motility is generated by spinal and brainstem circuitries, and the emergence of complex and variable GMs denotes the onset of supraspinal modulation of this spinal and brainstem activity. (Pediatr Res 63: 191-195, 2008)
\end{abstract}

$\mathrm{T}$ he impressive continuity of movement patterns from prenatal to postnatal life is not limited to specific patterns, such as isolated movements of arms, legs, head, and general movements (GMs). The continuity may also hold true for the quality of GMs. GMs with an abnormal quality present before birth and persisting to 3 mo postterm age are related to an adverse neurologic outcome at 1 year of age (1). The quality of GMs in preterm neonates has also been demonstrated to be a promising factor in finding the population at high risk of an adverse neurologic outcome at 1 year and school age (2-4).

The various specific movement patterns have emerged as early as 15 wk postmenstrual age (PMA) and can be recognized throughout later stages of pregnancy and during the first months after birth (5). The most frequently occurring specific movement patterns of the fetus and young infant are the GMs (5). GMs are movements in which all parts of the body participate. During the past decade, it became increasingly clear that the quality of GMs reflects the integrity of the young

Received March 14, 2007; accepted September 13, 2007.

Correspondence: Johanna I. P. de Vries, M.D., Ph.D., Institute for Fundamental and Clinical Human Movement Sciences, Department of Obstetrics and Gynaecology, VU University Medical Centre, Postbox 7057, 1007 MB Amsterdam, The Netherlands; e-mail: jip.devries@vumc.nl nervous system $(3,4)$. Normal GMs are characterized by variation, complexity, and fluency $(2,6)$. These characteristics disappear when movements become abnormal.

Relatively little is known about the expression of neurologic dysfunction in the early phases of pregnancy. Some case reports suggested that neural dysfunction in the first trimester of pregnancy may induce changes in the differentiation and the qualitative and quantitative performance of specific movement patterns. For example, deviances in motor development during the first trimester have been reported for acquired disorders such as spontaneous abortion (7) or exposure to maternal type 1 diabetes (8) and in fetuses with congenital anomalies such as anencephaly (9) and fetal akinesia deformation sequence $(10,11)$. However, how the various movement patterns develop from their first appearance onward has not been fully investigated.

Previously, fetal motor activity was studied mostly by transabdominal sonography with 5.0-MHz transducers. The introduction of the transvaginal transducer, which allows a closer approach to the embryo and fetus and thus the application of higher emission frequencies leading to better resolution, has opened new possibilities in the study of the development of fetal movements during the earliest stages of motor development.

The present study aims at elucidating the emergence of GMs. From transabdominal sonographic examinations, it is known that the first movements can be visualized between 7 and 8.5 wk PMA. These movements have been called "just discernible movements" and have been described as a slow and small shifting of the fetal contours lasting $1 / 2$ to $2 \mathrm{~s}$, usually as a single event (5). Thereafter, it was described that these movements disappeared and GMs emerged. But how these just discernible movements develop into GMs is not known. Such knowledge of typical development is a prerequisite for the understanding of deviant development during early ontogeny. In our study, we paid special attention to the movement characteristics, which are most typical for normal GMs, i.e. variation in movement speed and amplitude and movement complexity (variation in participating body parts and movement direction).

With this specific focus in mind, we addressed, based on transvaginal ultrasound recordings performed between 7 and

Abbreviations: GMs, general movements; PMA, postmenstrual age; SB, sideways bending 
13 wk PMA in a population of fetuses of uncomplicated pregnancies the following:

Precise descriptions of earliest motor activity

Emergence of earliest motor activity

Evaluation of quality by describing the variation in movement speed, amplitude, and complexity (in sonographic terms, movement complexity is reflected by variation in participating body parts and movement directions)

Quantitative development

\section{METHODS}

Subjects. Eighteen women pregnant by IVF were invited to participate after receiving a detailed explanation of the aim of the investigation and after providing written informed consent. The study was approved by the medical ethical committee of the VU University Medical Centre. The women underwent regular early sonographic examinations confirming the exact known gestational ages. All women were healthy, although two were euthyroid receiving hormone supplementation.

Measurements. Six continuous real-time ultrasound observations of 15 min between $7 \mathrm{wk}+0 \mathrm{~d}$ and $12 \mathrm{wk}+6 \mathrm{~d}$ PMA were planned per women. The planned inclusion of women was three women twice weekly between 7 and $9 \mathrm{wk}$, three twice weekly between 10 and $13 \mathrm{wk}$, and a further three weekly between 7 and 13 wk. It turned out not to be easy for the women in the first group to come twice weekly on such a short notice. Therefore, the third group, which was assessed only once weekly, was increased to six. As a result, 18 women participated in the study, and 73 observations were made, 37 registrations before $10 \mathrm{wk}+0 \mathrm{~d}$ and 36 thereafter. This resulted in a 1095 -min recording with a 343 -s $(0.5 \%)$ loss caused by maternal movements.

The duration was chosen because before $20 \mathrm{wk}$ gestational age, the longest pause in fetal motility lasts $13 \mathrm{~min}$ (12). The regions of interest [head, trunk, rump, and extremities (at least one upper and one lower)] were determined in a pilot study of singleton examinations in another 18 women before study onset. It turned out that at 7-9 wk, the most favorable plane was frontal/ coronal and at 9-13 wk, the longitudinal/mid-parasagittal section. The women were instructed to minimize coughing or laughing to obtain a continuous observation of the regions of interest of the fetus.

All examinations were performed by one investigator (A.B.L.) using the Aloka Pro Sound SDD 4000 equipped with a multifrequency $(3.8-7.5 \mathrm{MHz})$ transvaginal probe. All assessments were videotaped on a Sony s-VHS Hi-Fi videocassette recorder SVO-9500 MDP and subsequently digitized for offline analyses. Off-line analyses were carried out by two of the investigators (A.B.L. and C.M.K.), and the classification of movement patterns was later discussed by the research team until a consensus was reached about the classification. Interobserver agreement on the presence of sideways bending (SB) and GMs was evaluated based on seven recordings made between 9 wk and $0 \mathrm{~d}$ and $10 \mathrm{wk}$ and $5 \mathrm{~d}$. The number of SB per recording varied from 0 to 19 and that of GMs from 0 to 7. Agreement of the two assessors (A.B.L. and C.M.K.) on the rate of occurrence of $\mathrm{SB}$ and GMs was very high (Spearman $\rho 1.00$ and 0.97 , respectively).

The first step in the analysis was the description of movement patterns: movement patterns could be either previously described movement patterns such as the GMs (5) or new patterns. The following step consisted of comparing the onset of the specific movement patterns as visualized with transvaginal sonography from the present study with the data obtained transabdominally in an earlier study (5). Next, movement quality was analyzed. The following variation parameters were distinguished for each single movement: 1) Variation in movement speed was scored as substantial variation, little variation (mainly slow or mainly quick movements), and no variation (consistently slow movements or consistently fast movements). 2) Variation in movement amplitude was also scored as substantial variation, little variation (mainly large variation or mainly small variation), and no variation (consistently small or consistently large variation). 3) Movement complexity was determined by analyzing the way in which the body parts participated in the movement. Here we took into account whether all body parts participated in the movement and whether the movement parts induced variation in movement direction. Here, a similar trichotomy in scoring was applied: substantial complexity, little complexity, and no complexity. Separately, the presence of a rotation during a GM was scored when the trunk changed position by more than 90 degrees.

The analysis focused on the description of emergence of various specific movement patterns and their quantitative and qualitative development. With the Friedman two-way analysis of variance, it was examined whether the medians of the incidence of GMs and SBs was similar or changed with increasing age. $p$ Values of $<0.05$ indicated statistically significant changes.

\section{RESULTS}

Classification of movement patterns observed between 7 and 13 wk PMA. The transvaginal ultrasound recordings resulted in the discovery of new specific movement patterns. The newly distinguished movement patterns are described in Table 1. Movement patterns, which we observed and which had been reported previously (5), were the GMs, startle, breathing, hiccup, isolated retroflexion head, isolated rotation of head, isolated anteflexion of the head, isolated arm movement (slow or twitch), isolated leg movement (slow or twitch), hand-face contact, jaw opening, sucking/swallowing, yawn, stretch, stepping (13), and rotation of the trunk during a GM (13).

Table 1. Classification of movement patterns between 7 and 12 wk PMA

$S B$ of the head consists of a single, slow, active lateral bending of the head, either to the left or right, usually lasting about $1 \mathrm{~s}$. The movement does not exhibit variation in speed, amplitude, participating body parts, or direction.

$S B$ of the rump consists of a single, slow, active lateral SB of the rump. When the movement emerges, it first is only short lasting, but with advancing development, the duration may increase to about $3 \mathrm{~s}$. The movement does not exhibit variation in speed, amplitude, participating body parts, or direction.

$S B$ of the head and rump consists of a single, slow, active lateral movement of the head and rump toward each other. Initially, the movements last only a short time, but with advancing development, movement duration may increase to $5 \mathrm{~s}$, with exceptional values of $>20 \mathrm{~s}$. The movement does not exhibit variation in speed, amplitude, participating body parts, or direction.

$S B$ of the head and rump in combination with arm activity consists of a single, slow, active lateral bending of the head and/or rump together with an active arm movement. The movement may be very short lasting, but with advancing age, movement duration may increase to $1 / 2 \mathrm{~min}$. The movement does not exhibit variation in direction, but a little variation in speed, amplitude, and body parts can be observed. Full arm extensions are not part of the movement pattern.

$S B$ of the head and rump in combination with leg activity consists of a single, slow, active lateral bending of the head and/or rump together with an active leg movement. The movement may be very short lasting, but with advancing age, movement duration may increase to a few seconds. The movement does not exhibit variation in direction, but a little variation in speed, amplitude, and participating body parts can be observed. Full leg extensions are not part of the movement pattern.

$S B$ of the head and rump in combination with activity of the arms and legs is an active lateral movement of the head and/or rump together with an active arm and leg movement. Initially, the movement is short lasting, but with advancing age, it may last for about $30 \mathrm{~s}$. The movement does not exhibit variation in direction, as only movements in a single direction are produced, but a little variation in speed, amplitude, and participating body parts (head, rump, arm, and leg movements) may be observed.

Slow extension of the spine: The young fetus may show isolated slow extension movements of the spine. The movement may be followed by passive movements of other parts of the body. The movement can be distinguished from hiccup by the absence of displacement of the diaphragm, chest, or abdomen.

Twitch spine: Twitching activity in the spinal region is defined as an abrupt movement originating near the spinal column. The twitch may be followed by passive movements of other parts of the body. 
To facilitate the comparison of the newly distinguished movements with the GMs, we reiterate the GM definition:

This category is applicable if the whole body is moved but no distinctive patterning or sequencing of the body parts can be recognized. When they first appear at 8 and $9 \mathrm{wk}$, they are slow and of limited amplitude. At 10-12 wk, general movements become forceful. Movements of the limbs, trunk, and head are rapid but smooth in appearance. The movements are of large amplitude, and therefore they frequently cause a shift in fetal position during this age period. After $12 \mathrm{wk}$, general movements become more variable in speed and amplitude. They may last from about 1 to 4 min but wax and wane during this period. However variable these movements are, they are always graceful in character (5).

In short, this means that GMs after the age of 12 wk PMA are characterized by variation in speed, amplitude, and complexity. The present study (between 7 and 13 wk) showed that the criteria of variation were fulfilled from 9 wk onward instead of $8 \mathrm{wk}$. We observed that GMs at early age often started abruptly with startle, hiccup, or twitching activity at the spine, but GMs could also start slowly. GM activity after an abrupt onset was fluent. During GMs, rotations of more and less than 90 degrees emerged from 9 wk onward.

Differentiation and emergence of specific movement patterns. The emergence of various specific movement patterns as obtained in this study by $3.8-7.5-\mathrm{MHz}$ transvaginal sonography is related to findings by transabdominal $3.5-\mathrm{MHz}$ sonography (Fig. 1). By $0.5 \mathrm{wk}$, new specific movement patterns appear. In the present study, the earliest motility was

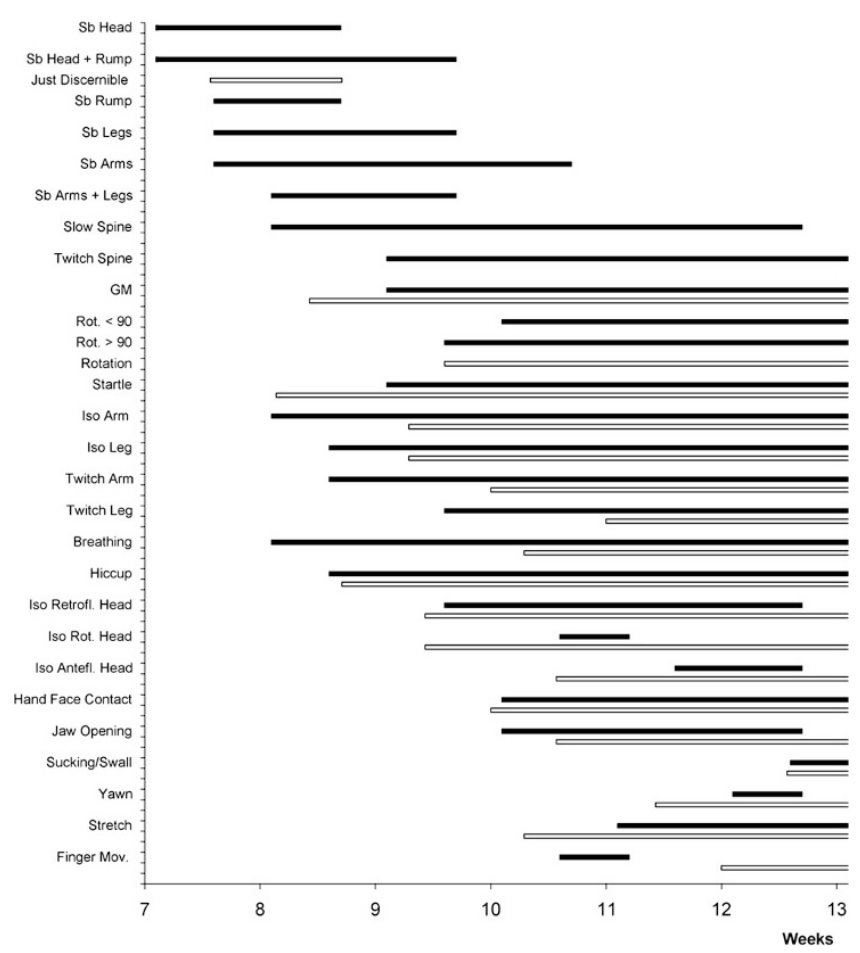

Figure 1. Emergence of specific fetal movement patterns in 18 fetuses between 7 and 13 wk PMA. The bars indicate age ranges during which the fetuses first showed a specific movement pattern. Black bars represent the data of the current study, the white bars, the data of de Vries et al. (5). Just discernible, just discernible movement; Rot, rotation; Iso, isolated; Retrofl, retroflexion; Antefl, anteflexion; Swall, swallowing. observed at $7 \mathrm{wk}$ and $2 \mathrm{~d}$, a few days earlier than previously noted. It consisted of small SB of the head, rump, or both. This means that the earliest movements of the fetus consist of slow, small, noncomplex, and isolated movements of the proximal parts of the body. At 7- $8.5 \mathrm{wk}$, these simple and stereotyped movements developed into movements in which also one or two arms or legs participated. But the movements at this age were still slow, small, simple, and stereotyped. In the period of 8-13 wk, a new specific movement pattern was also distinguished: the slow or abrupt extension movements of the spine.

In comparison with the older data, we found that GMs emerge $1 \mathrm{wk}$ later ( $9 \mathrm{wk}$ ), isolated arm and leg movements 1 wk earlier, and abrupt performed isolated arm and leg movements 2 wk earlier.

Quality of specific movement patterns: the emergence of movement variation and complexity. The first movements of the fetus consisted of movements of the head and rump. But a couple of days after the emergence of motility in the proximal body parts, the limbs also started to participate in fetal motility (Fig. 1). GMs, which consist of movements in which all parts of the body participate, emerge at the age of 9-10 wk. In 15 recordings, GMs and SB of the head and trunk coexisted.

The variation in the performance of the SB is presented in Table 2 . SB between $7+0$ and $8+2$ wk did not show any variation in speed and amplitude; the movements were consistently slow and small. Between $8+3$ and $9+6 \mathrm{wk}$, some degree of variation in speed and amplitude emerged to virtually disappear again thereafter. It should be noted, however, that SBs are rare beyond the age of $10 \mathrm{wk}$. Likewise, the emergence of variation in GM performance was studied (Table 3). Between $9+0$ and $9+2 \mathrm{wk}$, the first few GMs in general show little variation in speed, amplitude, and direction. Thereafter, i.e. from $9+3$ wk onward, the majority of GMs show a substantial degree of variation in speed, amplitude, participating body parts, and direction.

Quantity of specific movement patterns. The quantitative analyses showed that a large interindividual variation existed in the quantity of SB and GMs. Despite the large variation, a significant transition in motor output could be observed between 8 and 10 wk: the incidence of GMs significantly increased $(p=0.006)$ and that of SB decreased $(p=0.01$; Fig. 2).

The incidence of the abrupt specific movement patterns of hiccup, twitching movements of the spine, and startles is depicted in Figure 3. Between 8 and 11 wk, hiccups were seen most frequently, whereas from 11 to $13 \mathrm{wk}$, the ranges of occurrence of hiccups and twitching movement of the spine more or less overlapped. Startles were present in the observation period at a consistently low incidence.

\section{DISCUSSION}

This study revealed new developmental milestones in the differentiation and quality of specific movement patterns in the fetus before 13 wk PMA. Our major finding is a transition in early fetal motor behavior at 9 wk PMA. Fetal motility starts at wk 7 with relatively simple and stereotype SB movements of the head or trunk with or without extremity movements. 
Table 2. The variation in the performance of $S B$

\begin{tabular}{|c|c|c|c|c|c|c|c|c|}
\hline \multirow{2}{*}{$\begin{array}{l}\text { Gestational } \\
\text { age }\end{array}$} & \multirow{2}{*}{$\begin{array}{c}\text { Fetus, } \\
\text { no./total }\end{array}$} & \multirow[b]{2}{*}{ SB, no. } & \multicolumn{3}{|c|}{ Variation in speed, $\%$} & \multicolumn{3}{|c|}{ Variation in amplitude, $\%$} \\
\hline & & & + & \pm & - & + & \pm & - \\
\hline 7 wk $0-2 \mathrm{~d}$ & $1 / 5$ & 15 & & & 100 & & & 100 \\
\hline 7 wk $3-6$ d & $9 / 9$ & 88 & & & 100 & & & 100 \\
\hline 8 wk $0-2 \mathrm{~d}$ & $6 / 6$ & 93 & & & 100 & & & 100 \\
\hline 8 wk 36 d & $6 / 6$ & 110 & & 25 & 75 & 1 & 25 & 74 \\
\hline 9 wk $0-2 \mathrm{~d}$ & $4 / 4$ & 44 & 5 & 30 & 66 & 7 & 27 & 66 \\
\hline 9 wk $3-6$ d & $4 / 7$ & 13 & 46 & 38 & 15 & 46 & 46 & 8 \\
\hline 10 wk $0-2 \mathrm{~d}$ & $3 / 7$ & 3 & & & 100 & & & 100 \\
\hline 10 wk $3-6 \mathrm{~d}$ & $3 / 7$ & 4 & & 25 & 75 & & 25 & 75 \\
\hline 11 wk $0-2 \mathrm{~d}$ & $0 / 5$ & 0 & & & & & & \\
\hline 11 wk $3-6 \mathrm{~d}$ & $2 / 4$ & 3 & & & 100 & & & 100 \\
\hline 12 wk $0-2 \mathrm{~d}$ & $2 / 7$ & 2 & & & 100 & & & 100 \\
\hline 12 wk $3-6 \mathrm{~d}$ & $1 / 5$ & 2 & & & 100 & & & 100 \\
\hline
\end{tabular}

Speed: + , substantial variation; \pm , little variation; - , no variation. Amplitude: + , substantial variation; \pm , little variation; - , no variation.

Table 3. Variation in the performance of GMs

\begin{tabular}{|c|c|c|c|c|c|c|c|c|c|c|c|c|c|}
\hline \multirow{2}{*}{$\begin{array}{c}\text { Gestational } \\
\text { age }\end{array}$} & \multirow{2}{*}{$\begin{array}{c}\text { Fetus, } \\
\text { no./total }\end{array}$} & \multirow[b]{2}{*}{ GM, no. } & \multicolumn{3}{|c|}{ Speed, \% } & \multicolumn{3}{|c|}{ Amplitude, \% } & \multicolumn{2}{|c|}{$\begin{array}{c}\text { Complexity } \\
\text { participating } \\
\text { body parts, \% }\end{array}$} & \multicolumn{3}{|c|}{$\begin{array}{l}\text { Complexity } \\
\text { direction, \% }\end{array}$} \\
\hline & & & + & \pm & - & + & \pm & - & NA & A & + & \pm & - \\
\hline 9 wk $0-2 \mathrm{~d}$ & $1 / 4$ & 7 & 29 & 71 & & 57 & 42 & & & 100 & 57 & 42 & \\
\hline 9 wk $3-6 \mathrm{~d}$ & $7 / 7$ & 38 & 66 & 29 & 5 & 66 & 29 & 5 & 8 & 92 & 66 & 29 & 5 \\
\hline 10 wk $0-2 \mathrm{~d}$ & $7 / 7$ & 37 & 78 & 19 & 3 & 81 & 19 & & 3 & 97 & 78 & 22 & \\
\hline 10 wk $3-6 \mathrm{~d}$ & $7 / 7$ & 38 & 92 & 8 & & 92 & 8 & & & 100 & 92 & 8 & \\
\hline 11 wk $0-2 \mathrm{~d}$ & $5 / 5$ & 25 & 80 & 16 & 4 & 84 & 12 & 4 & 16 & 84 & 84 & 12 & 4 \\
\hline 11 wk $3-6 \mathrm{~d}$ & $4 / 4$ & 21 & 62 & 38 & & 62 & 38 & & & 100 & 62 & 38 & \\
\hline 12 wk $0-2 \mathrm{~d}$ & $7 / 7$ & 44 & 66 & 23 & 10 & 66 & 25 & 10 & 14 & 86 & 64 & 27 & 9 \\
\hline 12 wk $3-6 \mathrm{~d}$ & $5 / 5$ & 34 & 71 & 24 & 6 & 71 & 24 & 6 & 18 & 82 & 71 & 24 & 6 \\
\hline $13+0$ & $1 / 1$ & 8 & 87 & 13 & & 87 & 13 & & 13 & 87 & 87 & 13 & \\
\hline
\end{tabular}

Speed: +, substantial variation; \pm , little variation; - , no variation. Amplitude: + , substantial variation; \pm , little variation; - , no variation. Participating body parts: NA, not all participating; A, all participating. Direction: + , substantial variation; \pm , little variation; - , no variation.

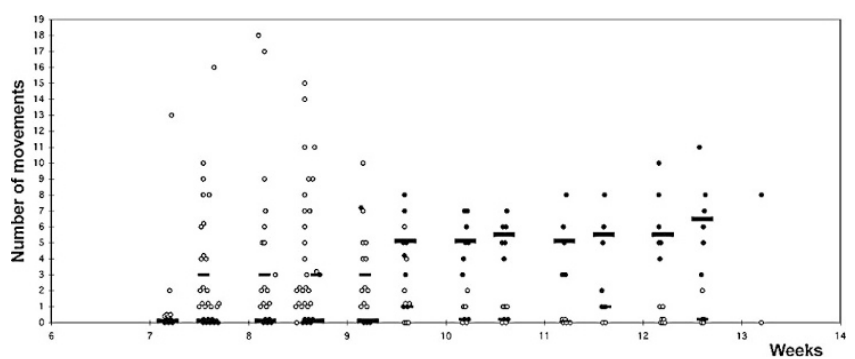

Figure 2. The number of SB and GMs per recording between 7 and $13 \mathrm{wk}$ PMA. $\bigcirc$, SB; $\bullet$, GMs; thick bar, median of general movement; thin bar, median of SB.

From wk 9 onward, these movements become more complex and variable, decrease in incidence, and are being replaced by GMs with variation and complexity. During a short period of time, stereotyped and complex movements coexist.

Methodologic considerations. Methodologically, our findings of an earlier emergence and new specific movement patterns and of more details of GM development can be explained by two factors. First, we now used high-resolution $3.8-7.5-\mathrm{MHz}$ transvaginal sonography in contrast to $3.5-\mathrm{MHz}$ transabdominal sonography used by de Vries et al. (5), which facilitated the description of more details of fetal motility. Second, the current study used shorter intervals of observations $(\leq 1 \mathrm{wk})$ than earlier studies and a precisely known gestational age thanks to IVF. The weaknesses of our protocol

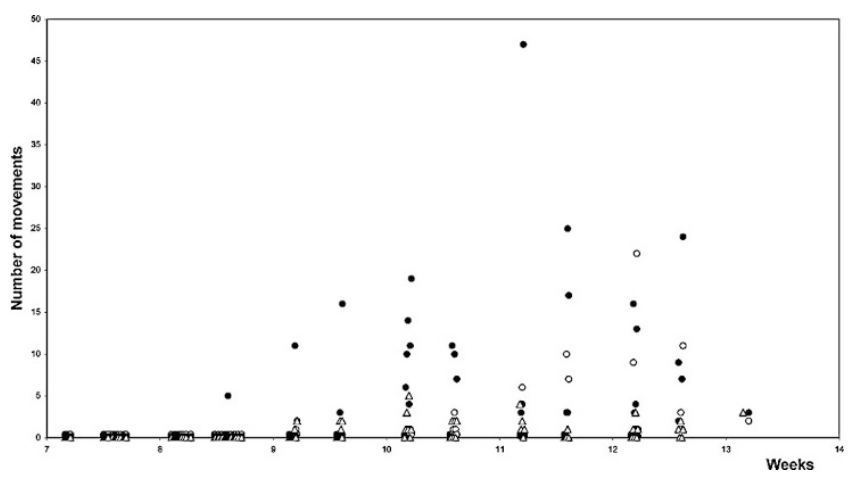

Figure 3. The number of hiccups, startles, and twitches of the spine per recording between 7 and 13 wk PMA. $\bigcirc$, twitch spine; $\bullet$, hiccup; $\Delta$, startle.

might be the observation time of only $15 \mathrm{~min}$. However, periods of rest longer than 13 min are not found in a normal population (12) and $15 \mathrm{~min}$ is a well accepted period of transvaginal imaging.

The better imaging techniques and the shorter assessment interval revealed that the first movements consist of SB of the head and/or rump followed by movements, which include slow and small extensions of an upper or lower extremity. This occurred within the period of 7-8.5 wk. Thus, these SBs develop in a time frame of days. SBs coexisted with GMs between 9 and $12 \mathrm{wk}$, whereas it was thought that they would 
disappear before the onset of GM (5). An additional new finding was that GM activity could also co-occur with slow and abrupt spine extensions. Due to the equipment with lower resolution at earlier times, the abrupt spine extensions had been classified as startles (5). The significance of the coexistence of simple and complex movement patterns is addressed later.

Ontogenetic considerations. Human fetal motility starts at 7.5 wk PMA. The motility consists of simple and stereotyped movements. Similar simple and stereotyped movements are reported as first movements in other species like fish (14), chicken (15), and rat (16). Wintrebert (14) in 1920 argued that the earliest forms of motility were purely myogenic, as sideways flexion of the head persisted after removal of the spinal cord. It is nowadays generally recognized that spontaneous activity is a major characteristic of the developing nervous system $(15,16)$. Thus, the earliest fetal movement activity is assumed to be generated by central pattern networks in the spinal cord and brainstem (17) and mediated by the immature muscle fibers of the myotomes (18). It is striking that the spontaneous movements as seen in the human fetuses of our study under physiologic circumstances emerge at the same gestational age as those elicited in human fetuses who were removed from the uterus under nonphysiologic circumstances (19).

GMs with their varied and complex character emerge from 9 wk PMA. Interestingly, the emergence of the variable and complex nature of GMs coincides with the emergence of an important transient cortical structure, the subplate $(20,21)$. The cortical subplate emerges around $9-10 \mathrm{wk}$ and functions as a temporary goal of afferent fibers from several areas (thalamus, basal forebrain, brainstem nuclei, contra- and ipsilateral hemispheres) heading for cortical destinations. The subplate is suggested to play a role in the guidance of some corticofugal pathways, and, as such, these early and transient cortical neuronal circuits may contribute to the emergence of fetal behavior. Whereas the SBs and extensions of the spine may be an expression of local spinal circuits, the rich variation in qualitative performance of the GMs strongly suggest a supraspinal influence. Thus, our study suggests that motor behavior brought about by spinal activity only and that which is the result of supraspinal modulation of spinal activity may coexist at $9-10 \mathrm{wk}$.

The present study has not only provided a timetable of the occurrence of specific movement patterns, but also the age after which GMs must exhibit a rich variation in speed, amplitude, and complexity. Both indices may have clinical application. In case of the absence of specific movement patterns beyond the age of $10 \mathrm{wk}$, PMA pathologic development should be strongly suspected. This has been shown for spontaneous abortion (7), anencephaly (9), and fetal akinesia deformation sequence $(10,11)$. In addition, we hypothesize that the absence of variable and complex movements beyond $10 \mathrm{wk}$ indicates the presence of supraspinal dysfunction caused, for instance, by a genetic disorder or an early insult to the brain.
This study indicates that the frequent occurrence of an abrupt onset of GMs between 9 and 13 wk is part of typical development. The presence of GMs with an abrupt onset has also been reported in healthy full-term neonates during sleep, in particular, during stages of sleep where cortical activity is less than during active wakefulness (22). Thus, the presence of abrupt-onset GMs in early fetal life may indicate that the modulating influence of supraspinal networks on the spinal GM networks fluctuates over time, allowing the occurrence of movements with an abrupt onset.

The present study indicates that from 9 to 10 wk PMA fetal GMs are characterized by movement variation and complexity. Studies at older ages indicated that the absence of movement variation and complexity are strong indicators of dysfunction of the supraspinal parts of the nervous system. Future studies should address the question of whether movement variation and complexity are equally powerful indicators of neurologic integrity of the young fetus.

\section{REFERENCES}

1. Sival DA, Visser GH, Prechtl HF 1992 The effect of intrauterine growth retardation on the quality of general movements in the human fetus. Early Hum Dev 28:119-132

2. Hadders-Algra M, Mavinkurve-Groothuis AM, Groen SE, Stremmelaar EF, Martijn A, Butcher PR 2004 Quality of general movements and the development of minor neurological dysfunction at toddler and school age. Clin Rehabil 18:287-299

3. Prechtl HF, Einspieler C, Cioni G, Bos AF, Ferrari F, Sontheimer D 1997 An early marker for neurological deficits after perinatal brain lesions. Lancet 349:1361-1363

4. Groen SE, de Blecourt AC, Postema K, Hadders-Algra M 2005 General movements in early infancy predict neuromotor development at 9 to 12 years of age. Dev Med Child Neurol 47:731-738

5. de Vries JI, Visser GH, Prechtl HF 1982 The emergence of fetal behaviour. I. Qualitative aspects. Early Hum Dev 7:301-322

6. Prechtl HF 1990 Qualitative changes of spontaneous movements in fetus and preterm infant are a marker of neurological dysfunction. Early Hum Dev 23:151-158

7. Ianniruberto A, Tajani E 1981 Ultrasonographic study of fetal movements. Semin Perinatol 5:175-181

8. Mulder EJ, Visser GH 1991 Growth and motor development in fetuses of women with type-1 diabetes. II. Emergence of specific movement patterns. Early Hum Dev 25:107-115

9. Shahidullah S, Hepper PG 1992 Abnormal fetal behaviour in first trimester spontaneous abortion. Eur J Obstet Gynecol Reprod Biol 45:181-184

10. Ajayi RA, Keen CE, Knott PD 1995 Ultrasound diagnosis of the Pena Shokeir phenotype at 14 weeks of pregnancy. Prenat Diagn 15:762-764

11. Paladini D, Tartaglione A, Agangi A, Foglia S, Martinelli P, Nappi C 2001 Pena-Shokeir phenotype with variable onset in three consecutive pregnancies. Ultrasound Obstet Gynecol 17:163-165

12. de Vries JI, Visser GH, Prechtl HF 1985 The emergence of fetal behaviour. II. Quantitative aspects. Early Hum Dev 12:99-120

13. de Vries JI, Visser GH, Prechtl HF 1984 Fetal motility in the first half of pregnancy. In: Prechtl HFR (ed) Continuity of Neural Functions from Prenatal to Postnatal Life (Clinics in Developing Medicine). Blackwell, Oxford, pp 46-64

14. Wintrebert $P$ [The rhythmic aneural contraction of the myotomes in the embryos of Selaciens. I. Observation of Scylliorhinus canicula, dogfish]. Arch Zool Exp Gen $192060: 221-459$

15. Bradley NS 2001 Age-related changes and condition-dependent modifications in distribution of limb movements during embryonic motility. J Neurophysiol 86:15111522

16. Bekoff A 2001 Development of motor behaviour in chick embryos. In: Kalverboer AF, Gramsbergen A (eds) Handbook of Brain and Behaviour in Human Development. Kluwer, Dordrecht, pp 429-55

17. Hadders-Algra M 2007 Putative neural substrate of normal and abnormal general movements. Neurosci Biobehav Rev [Epub ahead of print]

18. Mastaglia LF 1981 Growth and development of the skeletal muscle. In: Davis JA, Dobbing J (eds) Scientific Foundations of Pediatrics, 2nd ed. William Heinemann Medical Books, London, pp 590-620

19. Hooker D 1952 The sequence in human fetal activity. In: The Prenatal Origin of Behavior. University of Kansas Press, Lawrence, pp 54-86

20. de Graaf-Peters VB, Hadders-Algra M 2006 Ontogeny of the human central nervous system: what is happening when? Early Hum Dev 82:257-266

21. Molliver ME, Kostovic I, van der Loos H 1973 The development of synapses in cerebral cortex of the human fetus. Brain Res 50:403-407

22. Hadders-Algra M, Nakae Y, Van Eykern LA, Klip-Van den Nieuwendijk AW, Prechtl HF 1993 The effect of behavioural state on general movements in healthy full-term newborns. A polymyographic study. Early Hum Dev 35:63-79 